\title{
Estimating Excitonic Effects in the Absorption Spectra of Solids: Problems and Insight from a Guided Iteration Scheme
}

\author{
Santiago Rigamonti, ${ }^{1,2, *}$ Silvana Botti, ${ }^{3,4,2}$ Valérie Veniard, ${ }^{5,2}$ Claudia Draxl, ${ }^{1,2}$ Lucia Reining,,${ }^{5, \dagger}$ and Francesco Sottile ${ }^{5,2}$ \\ ${ }^{1}$ Humboldt-Universität zu Berlin, Institut für Physik and IRIS Adlershof, 12489 Berlin, Germany \\ ${ }^{2}$ European Theoretical Spectroscopy Facility (ETSF) \\ ${ }^{3}$ Institut Lumière Matière, UMR5306 Université Lyon 1-CNRS, Université de Lyon, F-69622 Villeurbanne Cedex, France \\ ${ }^{4}$ Friedrich-Schiller Universität Jena, Institut für Festkörpertheorie und -optik, Max-Wien-Platz 1, 07743 Jena, Germany \\ ${ }^{5}$ Laboratoire des Solides Irradiés, École Polytechnique, CNRS, CEA-DSM, F-91128 Palaiseau, France
}

(Received 28 November 2014; published 7 April 2015)

\begin{abstract}
A major obstacle for computing optical spectra of solids is the lack of reliable approximations for capturing excitonic effects within time-dependent density functional theory. We show that the accurate prediction of strongly bound electron-hole pairs within this framework using simple approximations is still a challenge and that available promising results have to be revisited. Deriving a set of analytical formulas we analyze and explain the difficulties. We deduce an alternative approximation from an iterative scheme guided by previously available knowledge, significantly improving the description of exciton binding energies. Finally, we show how one can "read" exciton binding energies from spectra determined in the random phase approximation, without any further calculation.
\end{abstract}

PACS numbers: 71.15.Qe, 71.10.-w, 71.35.-y, 78.20.Ci

The response of materials to an electromagnetic field is a key to many properties and applications. In the frequency range from infrared to ultraviolet, the optical properties determine the color of materials, their ability to absorb sunlight, and much more. They lay the ground for nondestructive spectroscopies, such as ellipsometry, that can tell us much about the electronic or atomic structure of materials. However, theoretical tools are needed that allow one to analyze, understand, and predict measured results and desired or undesired properties. These tools should be reliable and versatile, but simple enough to be applicable to systems of fundamental or technological interest, which are often rather complex. One of the major challenges is to design approximations for the ab initio calculation of optical spectra of extended systems such as solids and liquids [1].

The state-of-the-art approach for the ab initio calculation of optical spectra consists in using the Kohn-Sham electronic structure coming from a density functional theory calculation as the starting point for a quasiparticle band structure calculation in the $G W$ approximation, and the subsequent solution of the Bethe-Salpeter equation (BSE) to account for the electron-hole interaction [1-5]. The scheme is successful; in particular, excitonic effects are well described. However, the calculations are computationally demanding, because of the two-particle (electron and hole) nature of the problem. Alternatively, time-dependent density functional theory (TDDFT) $[1,6,7]$ formulates the response in terms of variations of local potentials that are functionals of the time-dependent density. This reduces the size of the problem, but raises the question of how to find a good approximation for the time-dependent exchange-correlation (xc) potential $v_{\mathrm{xc}}$ and its first derivative, the xc kernel $f_{\mathrm{xc}}\left(\mathbf{r}, \mathbf{r}^{\prime}, t-t^{\prime}\right)=\delta v_{\mathrm{xc}}(\mathbf{r}, t) / \delta n\left(\mathbf{r}^{\prime}, t^{\prime}\right)$, where $n$ is the time-dependent electron density. Some simple but widely used approximations such as the adiabatic local density approximation $[8,9]$, which are often successful for finite systems and for electron energy-loss spectra, yield disappointing results similar to the random phase approximation $\left(f_{\mathrm{xc}}=0\right)[7,10]$ for absorption spectra of solids.

Many works, e.g., Refs. [11-16], try to overcome this problem. A class of successful kernels has been derived from the BSE [17-22]. The nanoquanta kernel [20-23] gives results close to $\mathrm{BSE}$ ones, but with a comparable computational cost, although suggestions for speedups have been made [24]. The long-range corrected (LRC) kernel $[23,25] f_{\mathrm{xc}}^{\mathrm{LRC}}=-\alpha / q^{2}$ with the correct divergence for small wave vectors $q$ is a simple scalar approximation of the nanoquanta kernel. $f_{\mathrm{xc}}^{\mathrm{LRC}}$, with $\alpha$ empirically determined from the static dielectric constant of the crystal [25], works well for continuum excitons in semiconductors [26-28]. However, it fails to reproduce bound excitons, unless $\alpha$ is set ad hoc to a much higher value than in Ref. [25]. In this case, a transition may appear within the quasiparticle gap [29-31], but with too high oscillator strength [29].

Alternatively, the so-called bootstrap (BO) kernel [32] also has the correct $1 / q^{2}$ behavior; the prefactor is determined self-consistently, and it goes beyond the scalar version. Promising results have been published [32,33] for continuum and bound excitons, and the exciton binding energies of a range of small- and large-gap semiconductors have been calculated $[34,35]$. However, the BO expression has not been derived, but rather justified by observations, and the predictive power of the approach has not yet been demonstrated. Indeed, as we will show below, the BO does not lead to reliable absorption spectra, sometimes not even qualitatively. 
The aim of this work is to elucidate the origin of the BO and of its shortcomings, in order to go beyond. We show that a BO-like expression can indeed be derived, but it is slightly different from the ad hoc (i.e., without derivation) one of Ref. [32] and it leads to improvements, in particular for exciton binding energies. The computational cost can be further significantly reduced thanks to simple analytical formulas. In particular one can "read" exciton binding energies from results obtained in the random-phase approximation (RPA), without any further calculation.

Optical spectra of solids are obtained from the imaginary part of the macroscopic dielectric function $\epsilon_{M}(\omega)$, which can be calculated from

$$
\epsilon_{M}(\omega)=\frac{1}{\epsilon_{00}^{-1}(\omega)}=1-v_{0} \bar{\chi}_{00}(\omega),
$$

where $\epsilon_{\mathbf{G G}^{\prime}}(\omega)$ is the $\mathbf{q} \rightarrow 0$ limit of the microscopic dielectric matrix $\epsilon_{\mathbf{G G}^{\prime}}(\mathbf{q}, \omega)$ in a basis of reciprocal lattice vectors. 00 indicates the head $\left(\mathbf{G}=\mathbf{G}^{\prime}=0\right)$ element of the matrix, $v_{0}$ is the long-range $(\mathbf{G}=0, \mathbf{q} \rightarrow 0)$ part of the Coulomb interaction, and $\bar{\chi}$, the linear density response to the total macroscopic classical potential [1], is obtained from the matrix (in $\mathbf{G}, \mathbf{G}^{\prime}$ ) Dyson equation

$$
\begin{gathered}
\bar{\chi}(\omega)=\bar{\chi}^{\mathrm{RPA}}(\omega)+\bar{\chi}^{\mathrm{RPA}}(\omega) f_{\mathrm{xc}}(\omega) \bar{\chi}(\omega), \\
\bar{\chi}^{\mathrm{RPA}}(\omega)=\chi^{0}(\omega)+\chi^{0}(\omega) \bar{v} \bar{\chi}^{\mathrm{RPA}}(\omega)
\end{gathered}
$$

with $\bar{v}$ the Coulomb interaction without the $\mathbf{G}=0$ component $v_{0}$, and $\chi^{0}$ the independent-particle response function. The RPA solution $\bar{\chi}^{\mathrm{RPA}}(\omega)$ includes crystal local field effects (LFE) through $\bar{v}$. Note that $\chi^{0}$ is in principle the Kohn-Sham independent-particle response function. However, here we build $\chi^{0}$ with quasiparticle energies, e.g., from a $G W$ calculation (see the Supplemental Material for details [36]). Hence $f_{\mathrm{xc}}$ does not have to simulate the gap opening with respect to the Kohn-Sham gap. This often adopted strategy for TDDFT in solids allows one to simplify the kernel significantly, and is used also for the BO kernel [32]. The latter is a static matrix (middle term below)

$$
f_{\mathrm{xc}, \mathbf{G G}^{\prime}}^{\mathrm{BO}}=\frac{\epsilon_{\mathbf{G G}^{\prime}}^{-1}(0) v_{\mathbf{G}^{\prime}}}{1-\epsilon_{00}^{\mathrm{RPA}}(0)} \rightarrow \frac{1}{\epsilon_{M}(0) \chi_{00}^{0}(0)} .
$$

Often one can consider just the head element $f_{\mathrm{xc}, 00}$ without altering results significantly. The $\mathrm{BO}$ kernel is then the last term of Eq. (4), and Eq. (2) for $\bar{\chi}_{00}$ is scalar [53]. For clarity, in the following we will work with scalar equations unless stated. We have performed a detailed study for a family of matrix kernels [54] and found that results for the full matrix $f_{\mathrm{xc}}^{\mathrm{BO}}$ are similar to the present scalar version. We hence drop the subscripts 0 and consider the head of $\bar{\chi}^{\mathrm{RPA}}$ and $f_{\mathrm{xc}}$.

In Ref. [32] the equivalent of Eqs. (1), (2), and (4) were iterated numerically to self-consistency. However, this can easily be avoided since Eqs. (1), (2), and (4) combine to a quadratic equation for $\epsilon_{M}(0)$ with two solutions

$$
\begin{aligned}
\epsilon_{M}(0)= & \frac{1}{2}\left(1+\frac{\bar{\chi}^{\mathrm{RPA}}}{\chi^{0}}-v \bar{\chi}^{\mathrm{RPA}}\right) \\
& \pm \sqrt{\frac{1}{4}\left(1+\frac{\bar{\chi}^{\mathrm{RPA}}}{\chi^{0}}-v \bar{\chi}^{\mathrm{RPA}}\right)^{2}-\frac{\bar{\chi}^{\mathrm{RPA}}}{\chi^{0}}}
\end{aligned}
$$

where all quantities are static. Only the solution with the plus $(+)$ sign is meaningful, since in the limit of strong screening and neglecting LFE it leads to the RPA solution $\epsilon_{M}^{\mathrm{RPA}} \rightarrow$ $1-v \chi_{00}^{0}$ as expected. The minus sign would lead to $\epsilon_{M} \rightarrow 1$. Given $\chi^{0}$ and $\bar{\chi}^{\mathrm{RPA}}$, the static $\epsilon_{M}(\omega=0)$ from Eq. (5) and hence $f_{\mathrm{xc}}^{\mathrm{BO}}$ from Eq. (4) are numbers that can be determined on a pocket calculator and then used in Eq. (2) to correct a given RPA spectrum for excitonic effects. We have checked that the converged iterative results and those of Eq. (5) are indistinguishable. The next order in the strong screening expansion of Eq. (5) yields $\epsilon_{M}(\omega=0)=\epsilon_{M}^{\mathrm{RPA}}(\omega=0)+1$, which agrees with the typical magnitude of excitonic effects on the dielectric constant of semiconductors (see, for example, Table I of Ref. [25]).

Bound excitons occur when $\operatorname{Im} \epsilon_{M}\left(\omega_{0}\right)$, and hence $\operatorname{Im} \bar{\chi}\left(\omega_{0}\right)$, is nonvanishing at energies $\omega_{0}$ within the quasiparticle gap, where $\chi^{0}$ and $\bar{\chi}^{\mathrm{RPA}}$ are real. Since the scalar $f_{\mathrm{xc}}$ in Eq. (4) is real, the imaginary part of Eq. (2) is [29]

$$
f_{\mathrm{xc}}=\frac{1}{\bar{\chi}^{\mathrm{RPA}}\left(\omega_{0}\right)} \text {. }
$$

With Eq. (4), the position $\omega_{0}$ of the first excitonic peak inside the gap is then the implicit solution of

$$
\bar{\chi}^{\mathrm{RPA}}\left(\omega_{0}\right)=\epsilon_{M}(0) \chi^{0}(0) .
$$

By plotting $\operatorname{Re} \epsilon_{M}^{\mathrm{RPA}}(\omega)$ and comparing to the static $1-v \epsilon_{M} \chi^{0}$ with $\epsilon_{M}$ from Eq. (5), one can hence read exciton binding energies from a RPA spectrum. For illustration, we show bulk silicon, LiF, and solid argon. The black solid lines in Fig. 1 show the real part of $\epsilon_{M}^{\mathrm{RPA}}(\omega)$ for the three materials (for computational details, see the Supplemental Material [36]); it is monotonically increasing within the quasiparticle gap. The value $\left[1-\epsilon_{M}(0) v \chi^{0}(0)\right]$ with $\epsilon_{M}$ calculated with Eq. (5) is given by the horizontal red dashed lines, and the red vertical lines indicate intersections, hence bound excitons.

In silicon no bound exciton is found because $\epsilon_{M}(0)$ is large. $\mathrm{LiF}$ and argon have a low dielectric constant and therefore exhibit a crossing below the gap. However, the exciton binding energies, given by the difference between the energy of the fundamental quasiparticle gap and the exciton peak, are only $0.05 \mathrm{eV}$ in $\mathrm{LiF}$ and $0.0 \mathrm{eV}$ in $\mathrm{Ar}$, much smaller than the experimental results (about 1.4 and $2.0 \mathrm{eV}$, respectively [55,56]), and in apparent contrast to Ref. [32]. The latter discrepancy cannot be explained with the use of Eq. (7), which is exact when the BO kernel is used. Let us therefore look at the spectra. Figure 2 shows our results of $\mathrm{BO}$ calculations for the imaginary parts of the macroscopic dielectric function for $\mathrm{Si}, \mathrm{LiF}$, and Ar. 

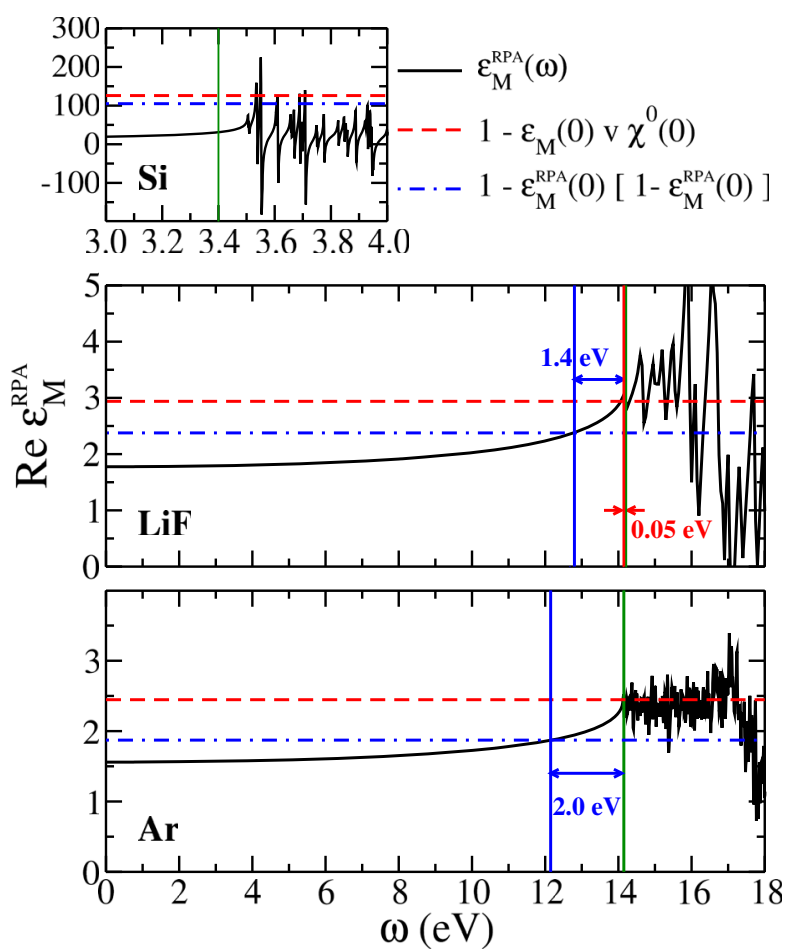

FIG. 1 (color online). Real part of $\epsilon_{M}^{\mathrm{RPA}}(\omega)$ for $\mathrm{Si}, \mathrm{LiF}$, and $\mathrm{Ar}$ (black solid line). Its crossing with the red dashed [blue dot-dashed] horizontal lines gives the exciton binding energy $\omega_{0}$ from Eq. (7) [Eq. (11)]. The green vertical line indicates the quasiparticle gap.

Red dashed curves stem from our TDDFT calculations with the BO kernel [Eq. (4)]. In silicon, like in Ref. [32], the kernel improves the spectrum with respect to quasiparticle RPA (QP-RPA) [57] by enhancing the first peak and inducing an overall transfer of oscillator strength to lower energies. However, the effect appears underestimated when compared to experiment [58] and to BSE results [25]. For silicon, the simple long-range $f_{\mathrm{xc}}^{\mathrm{LRC}}=\alpha / q^{2}$ is sufficient [23]. However, the prefactor $\alpha_{\mathrm{BO}}$ from the BO kernel defined in Eq. (4) is only $\alpha=-0.1$, too weak compared to the optimal value $\alpha=-0.2$ [25], which explains why the effect is not strong enough.

In $\mathrm{LiF}$ and $\mathrm{Ar}$, our $\mathrm{BO}$ spectra confirm the weak exciton binding energies obtained from Eq. (7). The spectral shapes look similar to the ones of Ref. [32]; however, the positions of the exciton peak differ and, for argon, the peak height from our BO is about half of that in Ref. [32]. As regards the peak position, we are not in contradiction with Ref. [32] since our quasiparticle gaps are close to experimental photoemission gaps. Instead, the quasiparticle gaps used in Ref. [32] are much smaller. This compensates the too small exciton binding energy and leads to seemingly good agreement with experimental optical spectra. We elaborate on this point in the Supplemental Material [36]. Additionally, it is important to note that the exciton binding energy is very sensitive to details, especially for strongly bound excitons. The reason is that the latter lie in a region where the real part of $\epsilon^{\mathrm{RPA}}(\omega)$ is very flat (see

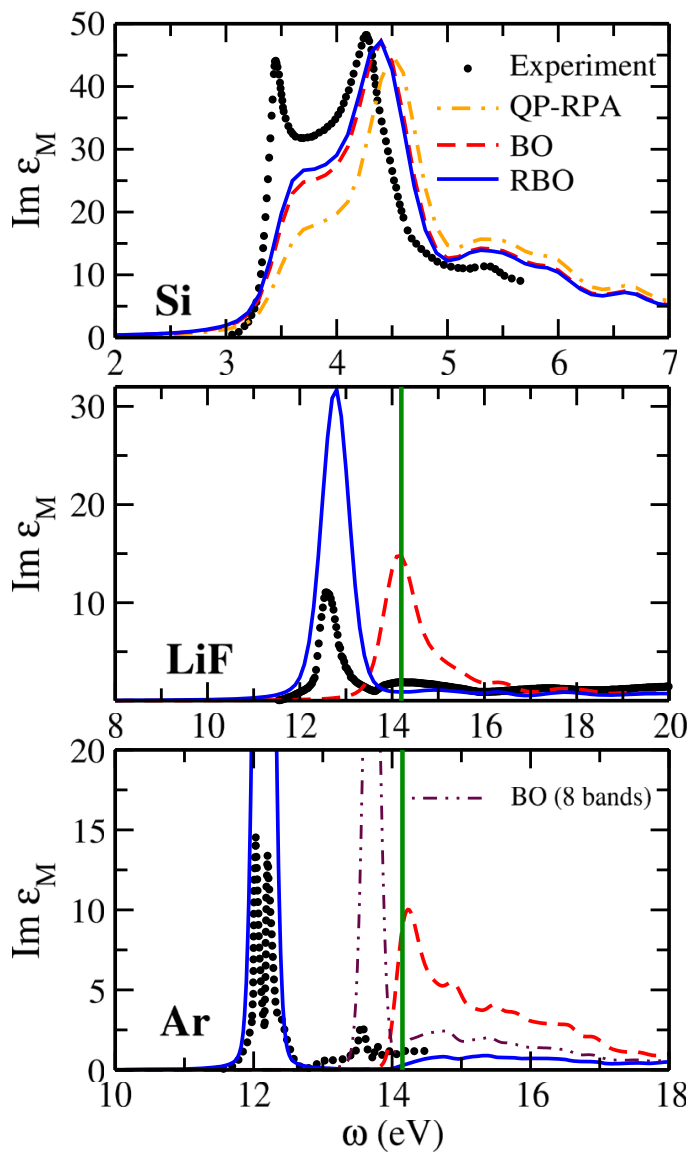

FIG. 2 (color online). Imaginary part of $\epsilon_{M}^{\mathrm{RPA}}(\omega)$ for $\mathrm{Si}, \mathrm{LiF}$, and Ar computed in various approximations. Experimental spectra are taken from Ref. [58] for Si, Ref. [55] for LiF, and Ref. [56] for Ar. The green vertical lines indicate the quasiparticle gap.

Fig. 1). A small change in $\chi^{0}(0)$ leads then to a large shift in the crossing point, and hence in the exciton binding energy. Such a small change in $\chi^{0}(0)$ can be due to a small change of the structure, or of computational ingredients like a pseudopotential or convergence parameters, and it can be amplified since the static dielectric constant enters the BO self-consistently. Indeed, the calculations for the BO kernel show a notable slow convergence with respect to both the LFE (i.e., number $N_{G}$ of $\mathbf{G}$ vectors) and the number of empty bands. The second issue is exemplified in Fig. 2 for the case of Ar: the brown double dot-dashed curve has been obtained with only 8 bands, versus 20 in the converged calculation (red dashed curve). The unconverged calculation exhibits a bound exciton with a binding energy of more than half an eV. Similarly, poorly converged calculations with respect to $N_{G}$ give also, for the case of argon, a BO spectrum with a slightly higher binding energy and a higher peak height than the converged result [54], much more similar to Ref. [32]. More generally, this explains why for argon or $\mathrm{LiF}$ one can easily obtain results that differ by an $\mathrm{eV}$ or more from others in the literature [34].

Once the calculations are settled, the results of the BO are hence disappointing. Let us therefore finally elucidate 
the origin of this kernel and indicate a possible improvement. We start from three assumptions: (A) We can take a static $f_{\mathrm{xc}}(\omega=0)$ in the optical range. (B) The static dielectric constant is larger than 1. (C) The static dielectric constant is not too different from the RPA one. These assumptions are based on previous knowledge from theory and experiment [assumption (B)], numerical results, e.g., of Bethe-Salpeter calculations [assumption (C)], and, most importantly [assumption (A)], insight from previous studies of long-range corrected kernels, e.g., Refs. [23,24,59]. The fact that $f_{\mathrm{xc}}$ should be proportional to the inverse dielectric constant $[23,25,60,61]$ has also been useful to guide the derivation, which we start by combining Eqs. (1) and (2):

$$
f_{\mathrm{xc}}=\frac{1}{\bar{\chi}^{\mathrm{RPA}}}-\frac{1}{\bar{\chi}}=\frac{1}{\bar{\chi}^{\mathrm{RPA}}}-\frac{v}{1-\epsilon_{M}} .
$$

If one had to make a guess for $f_{\mathrm{xc}}$ and iterate Eqs. (1), (2), and (8), one would of course get the same $f_{\mathrm{xc}}$ back, however absurd it might be. The trick of a BO-like approach is to make an approximation in one of the equations, such that they are no longer equivalent. At first sight this should not lead to any advantage: how could an approximation be better than the exact formula? However, by choosing the approximation carefully one can feed information. In that case, iteration of the (now no longer equivalent) equations may indeed define the three unknowns $f_{\mathrm{xc}}, \bar{\chi}$, and $\epsilon_{M}$. We will call this procedure "guided iteration." We will first use condition (A) to this aim: a static kernel can be determined from the equations at $\omega=0$ alone. In that limit, hypothesis (B) is generally valid, and we can use it to expand the $1 / \bar{\chi}$ term in Eq. (8) to leading order in $1 / \epsilon_{M}$

$$
\frac{1}{\bar{\chi}}=\frac{v}{1-\epsilon_{M}} \approx-\frac{v}{\epsilon_{M}} \approx-\frac{v}{\epsilon_{M}^{\mathrm{RPA}}},
$$

where we have used hypothesis $(C)$ in order to obtain the last expression. This finally leads to

$$
f_{\mathrm{xc}}^{\mathrm{RBO}} \approx \frac{1}{\epsilon_{M}^{\mathrm{RPA}} \bar{\chi}^{\mathrm{RPA}}},
$$

which we call the RBO (RPA bootstrap). The RBO is close to BO which appears in Eq. (4), but there is no self-consistency condition. The blue curves in Fig. 2 are obtained using Eq. (10). In silicon, the improvement with respect to the RPA result is close to that of the original BO (red dashed curve). Changes are noticeable in $\mathrm{LiF}$ and argon, where now the peak position is close to the experimental one [62]. Compared to experiment there is still too much spectral weight on these peaks. This is to be expected, because the two kernels behave like the LRC kernel. It is indeed known [29] that one can tune $\alpha$ to reproduce the exciton binding energy, but at the price of too much oscillator strength. To cure this problem, one may have to introduce a frequency dependence that is able to distribute spectral weight over the whole Rydberg series; this is however beyond the scope of the present work. Here, we focus on the exciton binding energy, which can now again be obtained from $\epsilon_{M}^{\mathrm{RPA}}(\omega)$ alone, using the modified prescription

$$
\bar{\chi}^{\mathrm{RPA}}\left(\omega_{0}\right)=\epsilon_{M}^{\mathrm{RPA}}(\omega=0) \bar{\chi}^{\mathrm{RPA}}(\omega=0) .
$$

This corresponds to the use of the blue horizontal dot-dashed line in Fig. 1. The exciton binding energies that we can read in this way, and which correspond of course to the peak positions given by the RBO in Fig. 2, are $2.0 \mathrm{eV}$ for argon and $1.4 \mathrm{eV}$ for $\mathrm{LiF}$, in excellent agreement with the experimental values of 2.0 and $1.43 \mathrm{eV}$, respectively.

In conclusion, starting from the so-called bootstrap kernel of TDDFT [32], we have derived very simple approaches to determine absorption spectra and to estimate exciton binding energies from RPA calculations alone. We have however shown that the boostrap kernel is not reliable for the determination of exciton binding energies, and that promising results in the literature are partially misleading. We have therefore derived a related kernel starting from a few physically meaningful assumptions. Numerical results confirm that the new kernel is more reliable. One may expect that this first derivation of a boostrap-like kernel could trigger new developments, but caution is called for: our guided iteration is not a systematic expansion that one might continue to obtain better and better results, since it intrinsically relies on the fact that an approximation is made by feeding knowledge. We stress again the importance of this approximation: Eqs. (1) and (2), and Eq. (8) are equivalent, though written in a different way; however, making an approximation on the second term of Eq. (8) [as in Eq. (9)] leads to a new formula, so breaking the otherwise tautological sequence Eqs. (1) and (2), and Eq. (8). The choice of a reasonable approximation (in this case the RBO) makes the method very effective in the description of the spectrum and, above all, for estimates of exciton binding energies. As we have shown and explained, these estimates are very sensitive, and a numerically precise agreement should not be overemphasized. Most importantly, we have shown that exciton binding energies can be obtained at literally zero cost, since we have introduced a way to read binding energies from RPA dielectric functions alone. This may be interesting especially for scientists outside the community of $a b$ initio calculations, including experimentalists, since it allows one to use the numerous already published RPA results, without the need of new calculations.

We are grateful for helpful discussions with S. Sharma and C. Ullrich. We acknowledge support by the French ANR (projects NT09-610745 and ANR-12-BS04-000102), the Einstein Foundation Berlin, and the DFG (Deutsche Forschungsgemeinschaft). Computer time was granted by GENCI (544).

*srigamonti@physik.hu-berlin.de

lucia.reining@polytechnique.edu

[1] G. Onida, L. Reining, and A. Rubio, Rev. Mod. Phys. 74, 601 (2002).

[2] W. Hanke and L. J. Sham, Phys. Rev. B 21, 4656 (1980). 
[3] S. Albrecht, L. Reining, R. Del Sole, and G. Onida, Phys. Rev. Lett. 80, 4510 (1998).

[4] L. X. Benedict, E. L. Shirley, and R. B. Bohn, Phys. Rev. Lett. 80, 4514 (1998).

[5] M. Rohlfing and S. G. Louie, Phys. Rev. Lett. 81, 2312 (1998).

[6] E. Runge and E. K. U. Gross, Phys. Rev. Lett. 52, 997 (1984).

[7] S. Botti, A. Schindlmayr, R. Del Sole, and L. Reining, Rep. Prog. Phys. 70, 357 (2007).

[8] E. Gross, J. Dobson, and M. Petersilka, in Density Functional Theory II, Topics in Current Chemistry, Vol. 181, edited by R. Nalewajski (Springer, Berlin, 1996), pp. 81-172.

[9] E. K. U. Gross and W. Kohn, Phys. Rev. Lett. 55, 2850 (1985).

[10] V. I. Gavrilenko and F. Bechstedt, Phys. Rev. B 55, 4343 (1997).

[11] V. U. Nazarov, G. Vignale, and Y.-C. Chang, Phys. Rev. Lett. 102, 113001 (2009).

[12] V. U. Nazarov and G. Vignale, Phys. Rev. Lett. 107, 216402 (2011).

[13] P. E. Trevisanutto, A. Terentjevs, L. A. Constantin, V. Olevano, and F. D. Sala, Phys. Rev. B 87, 205143 (2013).

[14] M. Hellgren and E. K. U. Gross, Phys. Rev. A 88, 052507 (2013).

[15] J. Paier, M. Marsman, and G. Kresse, Phys. Rev. B 78, 121201 (2008).

[16] P. Ghosez, X. Gonze, and R. W. Godby, Phys. Rev. B 56, 12811 (1997).

[17] R. Stubner, I. V. Tokatly, and O. Pankratov, Phys. Rev. B 70, 245119 (2004).

[18] U. von Barth, N. E. Dahlen, R. van Leeuwen, and G. Stefanucci, Phys. Rev. B 72, 235109 (2005).

[19] F. Bruneval, F. Sottile, V. Olevano, R. Del Sole, and L. Reining, Phys. Rev. Lett. 94, 186402 (2005).

[20] F. Sottile, V. Olevano, and L. Reining, Phys. Rev. Lett. 91, 056402 (2003).

[21] G. Adragna, R. Del Sole, and A. Marini, Phys. Rev. B 68, 165108 (2003).

[22] A. Marini, R. Del Sole, and A. Rubio, Phys. Rev. Lett. 91, 256402 (2003).

[23] L. Reining, V. Olevano, A. Rubio, and G. Onida, Phys. Rev. Lett. 88, 066404 (2002).

[24] F. Sottile, M. Marsili, V. Olevano, and L. Reining, Phys. Rev. B 76, 161103 (2007).

[25] S. Botti, F. Sottile, N. Vast, V. Olevano, L. Reining, H.-C. Weissker, A. Rubio, G. Onida, R. Del Sole, and R. W. Godby, Phys. Rev. B 69, 155112 (2004).

[26] W. Wełnic, S. Botti, L. Reining, and M. Wuttig, Phys. Rev. Lett. 98, 236403 (2007).

[27] G.-X. Zhang, A. Tkatchenko, J. Paier, H. Appel, and M. Scheffler, Phys. Rev. Lett. 107, 245501 (2011).

[28] H. Hübener, E. Luppi, and V. Véniard, Phys. Rev. B 83, 115205 (2011).

[29] F. Sottile, K. Karlsson, L. Reining, and F. Aryasetiawan, Phys. Rev. B 68, 205112 (2003).

[30] V. Turkowski and C. A. Ullrich, Phys. Rev. B 77, 075204 (2008).

[31] V. Turkowski, A. Leonardo, and C. A. Ullrich, Phys. Rev. B 79, 233201 (2009).

[32] S. Sharma, J. K. Dewhurst, A. Sanna, and E. K. U. Gross, Phys. Rev. Lett. 107, 186401 (2011).
[33] S. Sharma, J. K. Dewhurst, A. Sanna, A. Rubio, and E. K. U. Gross, New J. Phys. 14, 053052 (2012).

[34] Z.-h. Yang and C. A. Ullrich, Phys. Rev. B 87, 195204 (2013).

[35] Z.-h. Yang, Y. Li, and C. A. Ullrich, J. Chem. Phys. 137, 014513 (2012).

[36] See Supplemental Material at http://link.aps.org/ supplemental/10.1103/PhysRevLett.114.146402, for the computational details and an analysis of the influence of the quasiparticle gap in the optical spectra, which includes Refs. [37-52].

[37] P. Hohenberg and W. Kohn, Phys. Rev. 136, B864 (1964).

[38] W. Kohn and L. J. Sham, Phys. Rev. 140, A1133 (1965).

[39] N. Troullier and J. L. Martins, Phys. Rev. B 43, 1993 (1991).

[40] Pseudopotentials for the ABINIT code, http://www.abinit.org/ downloads/psp-links/psp-links/lda_tm.

[41] A. Gulans, S. Kontur, C. Meisenbichler, D. Nabok, P. Pavone, S. Rigamonti, S. Sagmeister, U. Werner, and C. Draxl, J. Phys. Condens. Matter 26, 363202 (2014).

[42] OPIUM pseudopotential generation program. http://opium .sourceforge.net/.

[43] J. P. Perdew and Y. Wang, Phys. Rev. B 33, 8800 (1986).

[44] G. B. Bachelet, D. R. Hamann, and M. Schlüter, Phys. Rev. B 26, 4199 (1982).

[45] J. A. Soininen and E. L. Shirley, Phys. Rev. B 61, 16423 (2000).

[46] H. J. Monkhorst and J. D. Pack, Phys. Rev. B 13, 5188 (1976).

[47] Numerical Data and Functional Relationships in Science and Technology, edited by K. H. Hellwege and O. Madelung (Springer, Berlin, 1982).

[48] M. Piacentini, D. W. Lynch, and C. G. Olson, Phys. Rev. B 13, 5530 (1976).

[49] M. Runne and G. Zimmerer, Nucl. Instrum. Methods Phys. Res., Sect. B 101, 156 (1995).

[50] L. Reining, V. Olevano, F. Sottile, S. Albrecht, and G. Onida, "The exc code" (unpublished).

[51] X. Gonze, J.-M. Beuken, R. Caracas, F. Detraux, M. Fuchs, G.-M. Rignanese, L. Sindic, M. Verstraete, G. Zerah, F. Jollet, M. Torrent, A. Roy, M. Mikami, P. Ghosez, J.-Y. Raty, and D. Allan, Comput. Mater. Sci. 25, 478 (2002).

[52] V. Olevano, L. Reining, and F. Sottile, "The dp code" (unpublished).

[53] Note that $\bar{\chi}_{00}^{\text {RPA }}$ still results from a matrix Eq. (3).

[54] S. Rigamonti, S. Botti, V. Véniard, C. Draxl, L. Reining, and F. Sottile (to be published).

[55] D. M. Roessler and W. C. Walker, J. Opt. Soc. Am. 57, 835 (1967).

[56] V. Saile, M. Skibowski, W. Steinmann, P. Gürtler, E. E. Koch, and A. Kozevnikov, Phys. Rev. Lett. 37, 305 (1976).

[57] QP-RPA stands for a RPA calculation with $\chi_{0}$ built with quasiparticle energies.

[58] P. Lautenschlager, M. Garriga, L. Vina, and M. Cardona, Phys. Rev. B 36, 4821 (1987).

[59] R. Del Sole, G. Adragna, V. Olevano, and L. Reining, Phys. Rev. B 67, 045207 (2003).

[60] E. G. Maksimov, I. I. Maxin, S. Y. Savrasov, and Y. A. Uspenski, J. Phys. Condens. Matter 1, 2493 (1989).

[61] M. A. L. Marques, J. Vidal, M. J. T. Oliveira, L. Reining, and S. Botti, Phys. Rev. B 83, 035119 (2011).

[62] Note that our calculations do not include the spin-orbit coupling, which is experimentally observed in Ar. 